\title{
The Equilibrium Structure of Hydrogen Peroxide
}

\author{
Joshua H. Baraban ${ }^{1, *}$ \\ Department of Chemistry, Ben-Gurion University of the Negev, Beer-Sheva, Israel, 84105 \\ P. Bryan Changala \\ JILA, National Institute of Standards and Technology and Department of Physics, University \\ of Colorado, Boulder, CO 80309, USA \\ John F. Stanton \\ Quantum Theory Project, Depts. of Chemistry and Physics, University of Florida, Gainesville, \\ FL 32611, USA
}

\begin{abstract}
The equilibrium structure of hydrogen peroxide is completely determined for the first time. Recent isotopically substituted data is combined with the results of rovibrational variational calculations to yield a complete semi-experimental structure, which is in excellent agreement with high level $a b$ initio calculated structures. In addition to numerically exact variational calculations, we also investigate the accuracy of approximate rovibrational predictions based on second order vibrational Møller-Plesset perturbation theory with curvilinear coordinates.
\end{abstract}

\section{Introduction}

Large amplitude motion is a commonly occurring but still insufficiently appreciated molecular behavior. A well-known example is the torsional tunneling found in the ground state of hydrogen peroxide [1]; despite extensive work on this fundamental reactive molecule, its equilibrium structure has never been determined precisely. One might have guessed that early attempts to derive the structural parameters of $\mathrm{H}_{2} \mathrm{O}_{2}$ from rotational spectroscopy were chiefly hampered by insufficient data (four independent coordinates but only three rotational constants $[2-8])$, but in fact the main difficulty was the very large difference between $r_{0}$ and $r_{e}$ for this decidedly floppy molecule. Significant progress was made on this

\footnotetext{
*Corresponding author

${ }^{1}$ Current address: Department of Chemistry and Biochemistry, University of Colorado, Boulder, CO 80309, USA
} 
problem by Koput $[9,10]$ but, without additional isotopic data, a complete structure could not be obtained. Sufficient information [11-15] now allows a complete semi-experimental equilibrium structure [16] to be determined.

The semi-experimental structure requires theoretical input from rovibrational calculations, for which we turn to numerically exact variational methods. These results also serve as a useful reference for assessing the performance of approximate rovibrational methods, in particular curvilinear coordinate vibrational second order Møller-Plesset perturbation theory (VMP2) [17-19] and a recently developed extension for $J>0$ rovibrational calculations [20]. The use of a curvilinear coordinate description makes this method applicable to molecules exhibiting large amplitude nuclear motion. Hydrogen peroxide, as the simplest torsionally isomerizing system, is therefore a particularly relevant benchmark.

\section{Methods}

Semi-experimental equilibrium rotational constants $\left(B_{e}^{s e}\right)$ were calculated by subtracting $a b$ initio rotation-vibration parameters $\left(B_{e}-B_{0}\right.$, frequently approximated in perturbation-theory-based methods as $\left.-\frac{1}{2} \sum_{i} \alpha_{i}^{B}\right)$ from the experimentally observed rotational constant values [11-15]. These rovibrational zero-point motion corrections were obtained with several treatments of the nuclear motion (second order vibrational perturbation theory (VPT2) [21], second-order vibrational Møller-Plesset theory (VMP2) [20], and variational) on potential energy surfaces (PES) using various levels of electronic structure theory [22, 23]. Variational calculations on the " $\mathrm{V}+\mathrm{C}+\mathrm{R}+\mathrm{H}$ " PES of Ref. [23] achieved the best agreement with the experimentally measured rovibrational levels, and so rotation-vibration parameters using this surface were employed for the structure fit. Electronic contributions to the rotational constants were also computed [24, 25] at frozen core CCSD(T)/aug-cc-pVTZ.

In addition to the PES, the VMP2 calculations require two major inputs: the internal vibrational coordinate system and the choice of molecule body-fixed frame embedding. For the former, we employed a curvilinear reaction path coordinate system, which is necessary to properly treat a large amplitude motion, multi-well system within the framework of VMP2. Multiple frame embeddings were used, including a standard Eckart frame tied to the planar trans transition state geometry and a moving Eckart frame tied to the instantaneous principal axis system along the torsional isomerization coordinate. We also investigated the effects of including the rotational degrees of freedom in the self-consistent field (SCF) portion of the calculation, making it an explicitly rovibrational SCF. We will refer to the perturbation theory results associated with this approach as "RVMP2". Further details of the VMP2/RVMP2 calculations are discussed in Appendix A. 
All calculations were performed with the CFOUR [26] and NITROGEN [27] program systems, using atomic masses.

\section{Results and Discussion}

\subsection{Equilibrium structure}

Figure 1 shows the semi-experimental equilibrium molecular structure, which is compared with an extrapolated ab initio structure $[28,29]$ in Table 1 . We wish to emphasize the large differences between the $r_{e}$ and $r_{0}$ structures reported in Table 1; as noted by Koput [10], it is critical to account for the effects of the large amplitude torsional motion. In general, the effects of zero point motion on the spectroscopic determination of molecular structures are under-appreciated, and in this particular case the vibration-rotation corrections amount to a few percent of the rotational constants. Perturbative quadruples have a surprisingly large effect on the torsional angle (nearly $0.3^{\circ}$ ) and the valence bond angle (almost $0.15^{\circ}$ ), presumably due to the interaction of the adjacent lone pairs.

Much of the debate in the literature over the structure of $\mathrm{H}_{2} \mathrm{O}_{2}$ has revolved around the choice of a reasonable value for $r_{\mathrm{OH}}$, so that the remaining parameters could be determined by the existing rotational constant data. Two predictions validated by the current work are a) that this $\left(r_{e}\right)$ bond length is more similar to that in water [30] $(0.95781(03) \AA,[31,32])$, than to that of the hydroxyl radical $(0.96966 \AA,[33])$, and b) that the $r_{0}$ value is approximately $0.967 \AA$. [5, 34] We rationalize this by noting that the hybridization in $\mathrm{H}_{2} \mathrm{O}_{2}$ is clearly more similar to $\mathrm{H}_{2} \mathrm{O}$ than to $\mathrm{OH}$.

Table 1: $\mathrm{H}_{2} \mathrm{O}_{2}$ geometries. Bond lengths in $\AA$, angles in degrees. The experimental structure is a semi-experimental equilibrium structure, $r_{e}^{s e}$, obtained by correcting experimental rotational constants for zero-point rotation-vibration effects calculated $a b$ initio.

\begin{tabular}{cccccc} 
Parameter & Calc. $^{a}$ & $r_{e}^{s e}$ & Ref. [10] & Ref. [23] & $r_{0}$ \\
\hline$r_{\mathrm{OH}}$ & 0.9623 & $0.9617(2)$ & $0.965(5)$ & 0.9628 & $0.9675(9)$ \\
$r_{\mathrm{OO}}$ & 1.4515 & $1.4524(3)$ & $1.452(5)$ & 1.4509 & $1.461(1)$ \\
$\angle \mathrm{OOH}$ & 100.07 & $99.76(6)$ & $100(1)$ & 100.09 & $100.07(25)$ \\
$\angle \mathrm{HOOH}$ & 113.17 & $113.6(3)$ & $112(1)$ & 112.81 & $119(1)$ \\
\hline
\end{tabular}

${ }^{a}$ Extrapolation of optimized all electron CCSD(T)/cc-pCVTZ-5Z geometries, supplemented by a higher order perturbative quadruples correction calculated with the pCVTZ basis set and the frozen core approximation. 


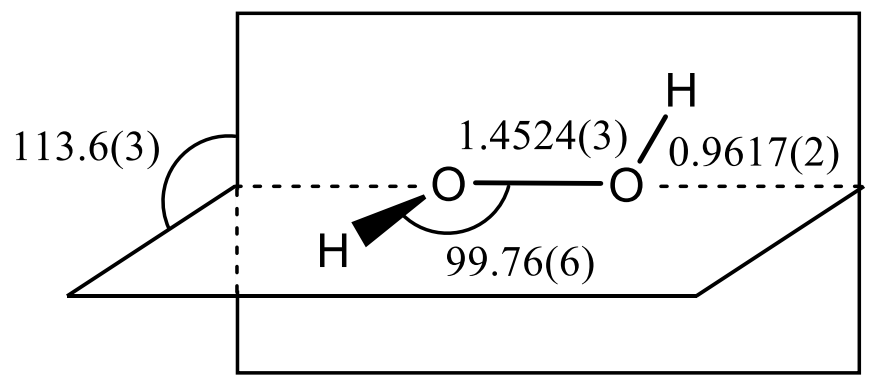

Figure 1: The molecular $r_{e}^{\text {se }}$ structure of $\mathrm{H}_{2} \mathrm{O}_{2}$. Bond lengths in $\AA$, angles in degrees.

\subsection{Comparing variational and VMP2 predictions}

The predicted energies for the small amplitude fundamental levels and torsional overtones are summarized in Tables 2 and 3, respectively. The root-mean-square difference between the perturbative VMP2 and reference variational energies is $0.32 \mathrm{~cm}^{-1}$ for the small amplitude fundamentals and $<0.01 \mathrm{~cm}^{-1}$ for the lowest 5 torsionally excited states. These errors are comparable to or smaller than the corresponding differences between the variational predictions and the observed values. In other words, the errors introduced by the VMP2 approximations are less important than errors in the Born-Oppenheimer potential energy surface. The small VMP2 errors, especially in the torsion overtone series, further indicate that the zeroth order picture of separable vibrations captures accurately all the important features of the vibrational dynamics. This success is entirely dependent on the use of the reaction path coordinate system. Other choices of coordinates would fare worse with this mean-field-based approach [19].

Table 2: Predicted fundamental frequencies of the five small amplitude vibrations of $\mathrm{H}_{2} \mathrm{O}_{2}$. All values are in $\mathrm{cm}^{-1}$.

\begin{tabular}{ccrrr} 
Mode & Description & VMP2 & Var. & Obs. $^{a}$ \\
\hline$\nu_{3}$ & OO stretch & 865.77 & 865.65 & 865.94 \\
$\nu_{6}$ & asym. bend & 1264.77 & 1264.78 & 1264.58 \\
$\nu_{2}$ & sym. bend & 1394.43 & 1394.36 & 1395.88 \\
$\nu_{1}$ & sym. OH stretch & 3609.73 & 3609.20 & 3609.8 \\
$\nu_{5}$ & asym. OH stretch & 3611.05 & 3610.49 & 3610.66 \\
\hline${ }^{a}$ Refs. $[35-38]$. & & &
\end{tabular}

Turning to rovibrational aspects, Table 4 compares the calculated zero-point vibrational corrections to rotational constants for a variety of methods against our variational benchmark. We focus on the predicted zero-point corrections, rather than the absolute ground state values, because it is the corrections that are needed to determine a semi-experimental equilibrium structure. Taking all three rotational 
Table 3: Predicted energies of the pure torsional levels, using the $(n, \tau)$ labeling convention. All values are in $\mathrm{cm}^{-1}$.

\begin{tabular}{lrrr}
$(n, \tau)$ & VMP2 & Var. & Obs. $^{a}$ \\
\hline$(0,1)$ & 0 & 0 & 0 \\
$(0,4)$ & 11.01 & 11.01 & 11.44 \\
$(1,1)$ & 256.41 & 256.40 & 254.55 \\
$(1,4)$ & 371.26 & 371.24 & 370.89 \\
$(2,1)$ & 570.32 & 570.32 & 569.74 \\
$(2,4)$ & 776.43 & 776.41 & 776.12 \\
\hline \multicolumn{2}{c}{ Ref. [8]. }
\end{tabular}

constants into account, the most accurate approximate method is the RVMP2 approach using the Eckart-LAM embedding. The significant difference between this and the standard VMP2 predictions with the same Eckart-LAM embedding is in the $A$ constant shift. This implicates torsion-rotation interactions about the $a$ axis as the primary difficulty, as expected. The improved accuracy of the RVMP2 vs. VMP2 predictions is a consequence of the fact that the torsional factor of the zeroth order RVMP2 wavefunction can adapt to angular momentum about the $a$-axis in the rotational wavefunction.

We also note that the (R)VMP2 calculations are uniformly more accurate than VPT2 predictions. The multi-well nature of the $\mathrm{H}_{2} \mathrm{O}_{2}$ PES and the resulting anharmonicity of the torsional degree of freedom are treated less than optimally by the zeroth order approximations of standard VPT2, namely those of rectilinear harmonic oscillator vibrations about a unique equilibrium geometry.

Table 4: Comparison of calculated zero-point vibrational shifts to rotational constants. All values are in MHz. The ground state rotational constants for variational, VMP2, and RVMP2 predictions were determined solely from $J=1$ energies, ignoring centrifugal distortion effects. "Eckart" refers to single-reference Eckart embedding using the trans transition state, and "Eckart-LAM" refers to the moving Eckart frame along the isomerization path. (See Appendix A for details.)

\begin{tabular}{cccccc} 
Parameter & Var. & VMP2 & VMP2 & RVMP2 & \\
(Eckart-LAM) & (Eckart-LAM) & VPT2 \\
\hline$A_{e}-A_{0}$ & 2346 & 2265 & 2419 & 2342 & 2731 \\
$B_{e}-B_{0}$ & 281 & 290 & 260 & 260 & 320 \\
$C_{e}-C_{0}$ & 507 & 520 & 530 & 529 & 445 \\
\hline
\end{tabular}

\section{Future Work}

The excellent performance of VMP2 for the pure vibrational energies leads us to believe that the corresponding rovibrational predictions, while good, can still 
be improved. As the primary difficulty is coupling of internal and total rotations, a more generalized zeroth order wavefunction that explicitly couples the torsional degree of freedom with rotations could potentially improve the treatment significantly.

Although the structure reported in Table 1 is complete and reasonably precise, a strong correlation (0.985) still exists between $r_{\mathrm{OH}}$ and $\angle \mathrm{OOH}$. Rotational constant data from ${ }^{18} \mathrm{O}$-substituted isotopologues is needed to definitively determine the structure of $\mathrm{H}_{2} \mathrm{O}_{2}$.

\section{Acknowledgements}

We thank J. T. Hougen for stimulating discussions on large amplitude motions of tetratomics, as well as for his extensive body of work on this subject, which is an inspiration to us all. P.B.C. is supported by an NSF Graduate Research Fellowship (Award No. DGE1144083), and J.F.S. would like to thank the U.S. National Science Foundation (Grant CHE-1361031), which also provided support for J.H.B. 
[1] J. T. Hougen, Summary of group theoretical results for microwave and infrared studies of $\mathrm{H}_{2} \mathrm{O}_{2}$, Canadian Journal of Physics 62 (12) (1984) 1392-1402.

[2] R. L. Redington, W. B. Olson, P. C. Cross, Studies of hydrogen peroxide: The infrared spectrum and the internal rotation problem, The Journal of Chemical Physics 36 (5) (1962) 1311-1326. arXiv:http://dx.doi.org/10.1063/1.1732733, doi:10.1063/1.1732733.

URL http://dx.doi.org/10.1063/1.1732733

[3] G. Khachkuruzov, I. Przhevalskii, Molecular constants of hydrogen peroxide. 4: Structural parameters, Optics and Spectroscopy 36 (1974) 172-174.

[4] R. H. Hunt, R. A. Leacock, C. W. Peters, K. T. Hecht, Internal-Rotation in Hydrogen Peroxide: The Far-Infrared Spectrum and the Determination of the Hindering Potential, The Journal of Chemical Physics 42 (6) (1965) 19311946. arXiv:http://dx.doi.org/10.1063/1.1696228, doi:10.1063/1.1696228.

URL http://dx.doi.org/10.1063/1.1696228

[5] D. Cremer, D. Christen, On the $r_{0^{-}}$and $r_{e^{-}}$-structures of $\mathrm{H}_{2} \mathrm{O}_{2}$, Journal of Molecular Spectroscopy $74 \quad$ (3) (1979) $480 \quad-482$. doi:https://doi.org/10.1016/0022-2852(79)90169-3.

URL http://www.sciencedirect.com/science/article/pii/0022285279901693

[6] P. A. Giguére, T. Srinivasan, The ground-state geometry of the $\mathrm{H}_{2} \mathrm{O}_{2}$ and $\mathrm{D}_{2} \mathrm{O}_{2}$ molecules, Journal of Molecular Spectroscopy 66 (1) (1977) $168-170$. doi:https://doi.org/10.1016/0022-2852(77)90331-9.

URL http://www.sciencedirect.com/science/article/pii/0022285277903319

[7] G. Pelz, K. Yamada, G. Winnewisser, Torsional Dependence of the Effective Rotational Constants of $\mathrm{H}_{2} \mathrm{O}_{2}$ and $\mathrm{H}_{2} \mathrm{~S}_{2}$, Journal of Molecular Spectroscopy 159 (2) (1993) 507 - 520. doi:https://doi.org/10.1006/jmsp.1993.1146.

URL http://www.sciencedirect.com/science/article/pii/S002228528371146X

[8] J.-M. Flaud, C. Camy-Peyret, J. W. C. Johns, B. Carli, The far infrared spectrum of $\mathrm{H}_{2} \mathrm{O}_{2}$. First observation of the staggering of the levels and determination of the cis barrier, The Journal of Chemical Physics 91 (3) (1989) 1504-1510. arXiv:http://dx.doi.org/10.1063/1.457110, doi:10.1063/1.457110. URL http://dx.doi.org/10.1063/1.457110

[9] J. Koput, On the $r_{0}^{\star}$ structure and the torsional potential function of hydrogen peroxide, Journal of Molecular Spectroscopy 115 (2) (1986) 438 441. doi:https://doi.org/10.1016/0022-2852(86)90058-5.

URL http://www.sciencedirect.com/science/article/pii/0022285286900585 
[10] J. Koput, An ab initio study on the equilibrium structure and torsional potential energy function of hydrogen peroxide, Chemical Physics Letters 236 (4) (1995) 516 - 520. doi:http://dx.doi.org/10.1016/0009-2614(95)00246Z.

URL http://www.sciencedirect.com/science/article/pii/000926149500246Z

[11] P. Helminger, W. C. Bowman, F. C. De Lucia, A study of the rotationaltorsional spectrum of hydrogen peroxide between 80 and $700 \mathrm{GHz}$, Journal of Molecular Spectroscopy 85 (1) (1981) 120-130.

[12] W. C. Bowman, F. C. D. Lucia, P. Helminger, The higher $\mathrm{K}_{-1}$ states of hydrogen peroxide, Journal of Molecular Spectroscopy 87 (2) (1981) 571 574. doi:https://doi.org/10.1016/0022-2852(81)90427-6.

URL http://www. sciencedirect.com/science/article/pii/0022285281904276

[13] J. J. Hillman, On the submillimeter spectrum of hydrogen peroxide, Journal of Molecular Spectroscopy 95 (1) (1982) 236 - 238. doi:https://doi.org/10.1016/0022-2852(82)90250-8.

URL http://www.sciencedirect.com/science/article/pii/0022285282902508

[14] J.-M. Flaud, J. W. Johns, Z. Lu, G. Winnewisser, H. Klein, The torsionrotation spectrum of $\mathrm{D}_{2} \mathrm{O}_{2}$, Canadian Journal of Physics 79 (2-3) (2001) 367374.

[15] D. Herberth, O. Baum, O. Pirali, P. Roy, S. Thorwirth, K. M. Yamada, S. Schlemmer, T. F. Giesen, Far infrared Fourier-transform spectroscopy of mono-deuterated hydrogen peroxide HOOD, Journal of Quantitative Spectroscopy and Radiative Transfer 113 (11) (2012) 1127 - 1133, three Leaders in Spectroscopy. doi:https://doi.org/10.1016/j.jqsrt.2012.02.035. URL http://www . sciencedirect.com/science/article/pii/S0022407312000994

[16] P. Pulay, W. Meyer, J. E. Boggs, Cubic force constants and equilibrium geometry of methane from Hartree-Fock and correlated wavefunctions, The Journal of Chemical Physics 68 (11) (1978) 5077-5085. arXiv:http://dx.doi.org/10.1063/1.435626, doi:10.1063/1.435626.

URL http://dx.doi.org/10.1063/1.435626

[17] L. S. Norris, M. A. Ratner, A. E. Roitberg, R. B. Gerber, Møller-Plesset perturbation theory applied to vibrational problems, The Journal of Chemical Physics 105 (24) (1996) 11261-11267. arXiv:http://dx.doi.org/10.1063/1.472922, doi:10.1063/1.472922.

URL http://dx.doi.org/10.1063/1.472922 
[18] O. Christiansen, Møller-Plesset perturbation theory for vibrational wave functions, The Journal of Chemical Physics 119 (12) (2003) 5773-5781. arXiv:http://dx.doi.org/10.1063/1.1601593, doi:10.1063/1.1601593.

URL http://dx.doi.org/10.1063/1.1601593

[19] D. Strobusch, C. Scheurer, Hierarchical expansion of the kinetic energy operator in curvilinear coordinates for the vibrational self-consistent field method, Journal of Chemical Physics 135 (2011) 124102.

[20] P. B. Changala, J. H. Baraban, Ab initio effective rotational and rovibrational Hamiltonians for non-rigid systems via curvilinear second order vibrational Møller-Plesset perturbation theory, The Journal of Chemical Physics 145 (17) (2016) 174106. doi:http://dx.doi.org/10.1063/1.4966234.

URL http://arxiv.org/abs/1609.00430

[21] I. M. Mills, Vibration-Rotation Structure in Asymmetric-and Symmetric-top molecules, in: K. N. Rao, C. W. Mathews (Eds.), Molecular Spectroscopy: Modern Research, Academic Press, 1972, Ch. 3.2.

[22] J. Koput, S. Carter, N. C. Handy, Potential energy surface and vibrational-rotational energy levels of hydrogen peroxide, The Journal of Physical Chemistry A 102 (31) (1998) 6325-6330. arXiv:http://dx.doi.org/10.1021/jp9812583, doi:10.1021/jp9812583.

URL http://dx.doi.org/10.1021/jp9812583

[23] P. Małyszek, J. Koput, Accurate ab initio potential energy surface and vibration-rotation energy levels of hydrogen peroxide, Journal of Computational Chemistry 34 (5) (2013) 337-345. doi:10.1002/jcc.23137.

URL http://dx.doi.org/10.1002/jcc. 23137

[24] W. H. Flygare, Magnetic interactions in molecules and an analysis of molecular electronic charge distribution from magnetic parameters, Chemical Reviews 74 (6) (1974) 653-687. arXiv:http://dx.doi.org/10.1021/cr60292a003, doi:10.1021/cr60292a003.

URL http://dx.doi.org/10.1021/cr60292a003

[25] C. Puzzarini, M. Heckert, J. Gauss, The accuracy of rotational constants predicted by high-level quantum-chemical calculations. I. molecules containing first-row atoms, The Journal of Chemical Physics 128 (19) (2008) 194108. arXiv:http://dx.doi.org/10.1063/1.2912941, doi:10.1063/1.2912941.

URL http://dx.doi.org/10.1063/1.2912941 
[26] J. F. Stanton, J. Gauss, M. E. Harding, P. G. Szalay, CFOUR, CoupledCluster techniques for Computational Chemistry, a quantum-chemical program package, with contributions from A.A. Auer, R.J. Bartlett, U. Benedikt, C. Berger, D.E. Bernholdt, Y.J. Bomble, L. Cheng, O. Christiansen, M. Heckert, O. Heun, C. Huber, T.-C. Jagau, D. Jonsson, J. Jusélius, K. Klein, W.J. Lauderdale, F. Lipparini, D.A. Matthews, T. Metzroth, L.A. Mück, D.P. O’Neill, D.R. Price, E. Prochnow, C. Puzzarini, K. Ruud, F. Schiffmann, W. Schwalbach, C. Simmons, S. Stopkowicz, A. Tajti, J. Vázquez, F. Wang, J.D. Watts and the integral packages MOLECULE (J. Almlöf and P.R. Taylor), PROPS (P.R. Taylor), ABACUS (T. Helgaker, H.J. Aa. Jensen, P. Jørgensen, and J. Olsen), and ECP routines by A. V. Mitin and C. van Wüllen. For the current version, see http://www.cfour.de.

[27] NITROGEN, Numerical and Iterative Techniques for Rovibronic Energies with General Internal Coordinates, a program written by P. B. Changala. http://www.colorado.edu/nitrogen.

[28] M. Heckert, M. Kállay, D. P. Tew, W. Klopper, J. Gauss, Basis-set extrapolation techniques for the accurate calculation of molecular equilibrium geometries using coupled-cluster theory, The Journal of Chemical Physics 125 (4) (2006) 044108. arXiv:http://dx.doi.org/10.1063/1.2217732, doi:10.1063/1.2217732.

URL http://dx.doi.org/10.1063/1.2217732

[29] C. Puzzarini, Extrapolation to the complete basis set limit of structural parameters: Comparison of different approaches, The Journal of Physical Chemistry A 113 (52) (2009) 14530-14535, pMID: 19799385. arXiv:http://dx.doi.org/10.1021/jp9040327, doi:10.1021/jp9040327.

URL http://dx.doi.org/10.1021/jp9040327

[30] W. R. Busing, H. A. Levy, Crystal and molecular structure of hydrogen peroxide: A neutron-diffraction study, The Journal of Chemical Physics 42 (9) (1965) 3054-3059. arXiv:http://dx.doi.org/10.1063/1.1696379, doi:10.1063/1.1696379.

URL http://dx.doi.org/10.1063/1.1696379

[31] A. Hoy, P. Bunker, A precise solution of the rotation bending Schrödinger equation for a triatomic molecule with application to the water molecule, Journal of Molecular Spectroscopy 74 (1) (1979) 1 - 8 . doi:http://dx.doi.org/10.1016/0022-2852(79)90019-5.

URL http://www.sciencedirect.com/science/article/pii/0022285279900195 
[32] A. G. Császár, G. Czakó, T. Furtenbacher, J. Tennyson, V. Szalay, S. V. Shirin, N. F. Zobov, O. L. Polyansky, On equilibrium structures of the water molecule, The Journal of Chemical Physics 122 (21) (2005) 214305. arXiv:http://dx.doi.org/10.1063/1.1924506, doi:10.1063/1.1924506.

URL http://dx.doi.org/10.1063/1.1924506

[33] K. P. Huber, G. Herzberg, Molecular structure and molecular spectra IV. Constants of diatomic molecules, Van Nostrand Reinhold Company, New York.

[34] D. Cremer, Theoretical determination of molecular structure and conformation. I. The role of basis set and correlation effects in calculations on hydrogen peroxide, The Journal of Chemical Physics 69 (10) (1978) 4440-4455. arXiv:http://dx.doi.org/10.1063/1.436434, doi:10.1063/1.436434.

URL http://dx.doi.org/10.1063/1.436434

[35] W. B. Olson, R. H. Hunt, B. W. Young, A. G. Maki, J. W. Brault, Rotational Constants of the Lowest Torsional Component (0G) of the Ground State and Lowest Torsional Component $(1 \mathrm{G})$ of the First Excited Torsional State of Hydrogen Peroxide, Journal of Molecular Spectroscopy 127 (1988) 12-34.

[36] C. Camy-Peyret, J.-M. Flaud, J. W. C. Johns, M. Noël, Torsion-Vibration Interaction in $\mathrm{H}_{2} \mathrm{O}_{2}$ : First High-Resolution Observation of $n u_{3}^{1}$, Journal of Molecular Spectroscopy 155 (1992) 84-104.

[37] W. B. Cook, R. H. Hunt, W. N. Shelton, F. A. Flaherty, Torsion-Rotation Energy Levels, Hindering Potential, and Inertial Parameters of the First Excited Vibrational State of the Antisymmetric O-H Stretch in Hydrogen Peroxide, Journal of Molecular Spectroscopy 171 (1995) 91-112.

[38] A. Perrin, A. Valentin, J.-M. Flaud, C. Camy-Peyret, L. Schriver, A. Schriver, P. Arcas, The 7.9- $\mu \mathrm{m}$ Band of Hydrogen Peroxide: Line Positions and Intensities, Journal of Molecular Spectroscopy 171 (1995) 358-373.

[39] J. T. Hougen, P. Bunker, J. Johns, The vibration-rotation problem in triatomic molecules allowing for a large-amplitude bending vibration, Journal of Molecular Spectroscopy 34 (1) (1970) 136-172.

\section{Appendix A. VMP2 details: coordinate system and frame embedding choices}

Appendix A.1. Internal coordinate system

To construct the internal coordinate system, we begin with the six standard curvilinear Z-matrix coordinates for a sequentially bonded tetratomic molecule 
$\vec{q}=\left(r_{\mathrm{OH}}, r_{\mathrm{OH}}^{\prime}, r_{\mathrm{OO}}, \theta_{\mathrm{OOH}}, \theta_{\mathrm{OOH}}^{\prime}, \tau_{\mathrm{HOOH}}\right)^{T}$. These are then used to define a reaction path coordinate system via the transformation [39]

$$
\vec{q}=\vec{r}(s)+\mathbf{R}(s) \vec{d} .
$$

The reaction path geometry in the original coordinates $\vec{q}$ is given by $\vec{r}$ as a function of the reaction (or isomerization) coordinate $s$. Displacements from the reaction path are given by the five local normal coordinates $\vec{d}=\left(d_{1}, \ldots, d_{5}\right)^{T}$, which are defined by the transformation matrix $\mathbf{R}$ at each point along the reaction path. The reaction path geometry $\vec{r}(s)$ is determined by covariant steepest descent starting from either of the two the planar (cis and trans) stationary points on the PES towards the two equivalent non-planar equilibrium geometries. Along the path, a projected GF normal mode calculation is performed to determine the $\mathbf{R}$ matrix defining the local normal coordinates, which are orthogonal to the path tangent. The primary benefit of the reaction path coordinate system is that it naturally interpolates between the normal mode coordinate systems of all four stationary points on the PES, achieving a high degree of separability in the vibrational Hamiltonian.

\section{Appendix A.2. Frame embedding and RVMP2}

The rotational VMP2 method we use here accounts for the rotational terms in the molecular kinetic energy operator perturbatively based on zeroth order wavefunctions calculated with the $J=0$ Hamiltonian. The perturbative approach works best if the rotation-vibration coupling is small. For molecules with a single, well-defined equilibrium geometry, applying the well known Eckart conditions minimizes this coupling near the equilibrium geometry. $\mathrm{H}_{2} \mathrm{O}_{2}$, however, undergoes large amplitude torsional motion and samples two non-planar local minima. Therefore, the simplest approach to frame embedding is to apply the Eckart conditions with respect to the planar trans transition state that connects these two wells. A more elaborate approach is to use a moving Eckart reference geometry tied to the large amplitude motion. In this case, the Eckart reference geometry corresponds to the instantaneous principal axis system geometry at each point along the isomerization reaction path.

As discussed in the main text, we can perform an even more sophisticated treatment by including the rotational degrees of freedom explicitly in the zeroth order SCF stage of the VMP2 calculation. That is, instead of using $J=0$ vibrational wavefunctions as the starting point for the rotational calculation, we allow the vibrational wavefunctions to adapt to a given rotational wavefunction (and vice versa) at the mean field level and then continue with standard second order perturbation theory corrections. We will term this explicit rovibrational approach RVMP2. 
Appendix A.3. Further technical details

The VMP2 calculations represent the rovibrational Hamiltonian using several many-body expansions. For the calculations reported here, the expansion orders were $\left(N_{V}, N_{G 1}, N_{G 2}, N_{U}, N_{V T}, N_{G r v}, N_{G r r}\right)=(5,4,4,3,3,4,4)$, including a dereferenced torsional coordinate [20]. Additionally, the virtual configuration space was constricted to a maximum of 8 quanta in both the dereferenced torsional mode and the sum of the excitations in the non-deferenced small amplitude modes. For the excited torsional state and rotational calculations, the configuration space was expanded to up to 20 quanta in the torsional mode. 